\title{
Reply to "circadian variation in acute myocardial infarction size: likely involvement of the melatonin and suprachiasmatic nuclei"
}

Heerajnarain Bulluck MBBS ${ }^{1,2,9}$, Jennifer Nicholas $\mathrm{PhD}^{3}$, Gabriele Crimi $\mathrm{MD}^{5}$, Steven K White MBBS ${ }^{1}$, Andrew J Ludman MD ${ }^{4}$, Silvia Pica MD ${ }^{6}$, Claudia Raineri $\mathrm{MD}^{5}$, Hector A Cabrera-Fuentes $\mathrm{PhD}^{8,9,10}$, Derek Yellon $\mathrm{PhD}^{1}$, Jose RodriguezPalomares $\mathrm{PhD}^{7}$, David Garcia-Dorado $\mathrm{PhD}^{7}$, Derek J Hausenloy $\mathrm{PhD}^{1,2,9,10}$

${ }^{1}$ The Hatter Cardiovascular Institute, Institute of Cardiovascular Science, University College London, UK

${ }^{2}$ The National Institute of Health Research University College London Hospitals Biomedical Research Centre, UK

${ }^{3}$ London School Hygiene and Tropical Medicine, London, UK

${ }^{4}$ Royal Devon and Exeter Hospital NHS Foundation Trust, Exeter, UK

${ }^{5}$ Struttura Complessa Cardiologia, Fondazione Istituto Di Ricovero e Cura a Carattere Scientifico (IRCCS) Policlinico San Matteo, Pavia, Italy

${ }^{6}$ Multimodality Cardiac Imaging Section, IRCCS Policlinico San Donato, Milan, Italy

${ }^{7}$ Cardiology Department, Vall d'Hebron Hospital, Universitat Autónoma de

Barcelona, Barcelona, Spain

${ }^{8}$ Institute of Biochemistry, Medical School, Justus-Liebig-University, Giessen, Germany

${ }^{9}$ National Heart Research Institute Singapore, National Heart Centre Singapore

${ }^{10}$ Cardiovascular and Metabolic Disorders Program, Duke-National University of Singapore

Short running title: Circadian variation in infarct size by CMR

Conflicts of interest: none

\section{Corresponding author:}

Professor Derek J Hausenloy

Cardiovascular \& Metabolic Diseases Program

Duke-NUS Graduate Medical School Singapore

8 College Road,

Singapore 169857

Tel +65 65166719

Email derek.hausenloy@duke-nus.edu.sg 
We read with great interest the letter by Dominguez-Rodriguez, Abreu-Gonzalez and Reiter regarding our article.[1] We thank the authors for pointing out that although the circadian "clock" genes are expressed by most tissues, the suprachiasmatic nuclei of the hypothalamus are predominantly responsible for their secretion and regulation in mammals and they also influence the expression of melatonin. Melatonin has numerous benefits as described in their letter but it is secreted in a circadian pattern. This may partly explain our findings of a circadian variation of myocardial infarction size, depending on the time of onset of symptoms. We admire their enthusiasm to champion melatonin as a promising cardioprotective agent against ischemia - reperfusion injury in the setting of ST-segment elevation myocardial infarction (STEMI).[2] Although the MARIA trial[2] was neutral for infarct size reduction, a post-hoc analysis found that those presenting within 2.5 hours of symptoms onset showed a significant reduction in myocardial infarct size.[3] This highlights the fact that careful patient selection is crucial to optimize the translation of promising cardioprotective therapies in the clinical setting.[4] Furthermore, around 50\% of STEMI patients treated by primary percutaneous coronary intervention develop microvascular obstruction[5] and therefore any cardioprotective therapy, even if administered via the intra-coronary route, would fail to reach the microcirculation in half of these patients. An alternative approach, which has not yet been studied so far, may be to combine low dose thrombolysis (to regain flow in the microcirculation as currently being investigated in the T-TIME trial - NCT02257294) with a promising cardioprotective therapy (to reach the ischemic myocytes and protect against lethal myocardial injury), in order to minimize reperfusion injury and improve outcomes in these patients.

\section{Acknowledgements}


Part of this work was supported by the British Heart Foundation (FS/10/039/28270), the Rosetrees Trust, and the National Institute for Health Research University College London Hospitals Biomedical Research Centre. HACF is funded by a Startup Grant of the "Excellence Cluster Cardio-Pulmonary System" (ECCPS) from the German Research Foundation (DFG, Bonn, Germany) and the "Peter und Traudl EngelhornStiftung" (Weilheim, Germany).

\section{References}

[1] Bulluck H, Nicholas J, Crimi G, White SK, Ludman AJ, Pica S, et al. Circadian variation in acute myocardial infarct size assessed by cardiovascular magnetic resonance in reperfused STEMI patients. International journal of cardiology. 2017;230:149-54.

[2] Dominguez-Rodriguez A, Abreu-Gonzalez P, de la Torre-Hernandez JM, Gonzalez-Gonzalez J, Garcia-Camarero T, Consuegra-Sanchez L, et al. Effect of intravenous and intracoronary melatonin as an adjunct to primary percutaneous coronary intervention for acute ST-elevation myocardial infarction: results of the Melatonin Adjunct in the acute myocaRdial Infarction treated with Angioplasty (MARIA) trial. J Pineal Res. 2016.

[3] Dominguez-Rodriguez A, Abreu-Gonzalez P, Reiter RJ. Melatonin as an Agent for Cardioprotection in Patients with ST-Elevation Myocardial Infarction and Short Ischaemic Time. Cardiovascular drugs and therapy / sponsored by the International Society of Cardiovascular Pharmacotherapy. 2017.

[4] Bulluck H, Yellon DM, Hausenloy DJ. Reducing myocardial infarct size: challenges and future opportunities. Heart. 2016;102:341-8.

[5] Bulluck H, Hausenloy DJ. Microvascular Obstruction: The Bane of Myocardial Reperfusion. Revista espanola de cardiologia. 2015;68:919-20. 